\title{
A low-mass stellar companion of the planet host star HD 75289^
}

\author{
M. Mugrauer ${ }^{1}$, R. Neuhäuser ${ }^{1}$, T. Mazeh ${ }^{2}$, J. Alves $^{3}$, and E. Guenther ${ }^{4}$ \\ 1 Astrophysikalisches Institut, Universität Jena, Schillergäßchen 2-3, 07745 Jena, Germany \\ e-mail: markus@astro.uni-jena.de \\ 2 Tel Aviv University, Tel Aviv 69978, Israel \\ ${ }^{3}$ European Southern Observatory, Karl-Schwarzschild-Str. 2, 85748 Garching, Germany \\ 4 Thüringer Landessternwarte Tautenburg, Sternwarte 5, 07778 Tautenburg, Germany
}

Received 2 April 2004 / Accepted 3 June 2004

\begin{abstract}
We report on the detection of a new low-mass stellar companion of HD 75289, a G0V star that harbors one known radial-velocity planet (Udry et al. 2000, A\&A, 356, 590). Comparing an image from 2MASS with an image we obtained with SofI at the ESO $3.58 \mathrm{~m}$ NTT three years later, we detected a co-moving companion located $21.465 \pm 0.023 \operatorname{arcsec}(621 \pm 10 \mathrm{AU}$ at $29 \mathrm{pc}$ ) east of HD 75289. A second SofI image taken 10 months later confirmed the common proper motion of HD 75289 B with its host star. The infrared spectrum and colors of the companion are consistent with an M 2 to M 5 main-sequence star at the distance of HD 75289. No further (sub)stellar companion down to $H=19$ mag could be detected. With the SofI detection limit we can rule out additional stellar companions beyond $140 \mathrm{AU}$ and substellar companions with masses $m \geq 0.050 M_{\odot}$ from $400 \mathrm{AU}$ up to $2000 \mathrm{AU}$.
\end{abstract}

Key words. stars: low-mass, brown dwarfs - stars: planetary systems

\section{Introduction}

More than 100 extrasolar planets have been discovered so far. Some of these planets have been found in multiple stellar systems. These planets are of particular interest, because they could provide some hints about the possible implications of stellar multiplicity for planet formation and for the stability and evolution of planet orbits. A first indication of such possible influence could be the apparent difference between the massperiod relation for planets in systems with only one star and that of planets in multiple stellar systems (Zucker \& Mazeh 2002). Furthermore Eggenberger et al. (2004) pointed out that planets orbiting in multiple stellar systems tend to have a very low eccentricity when their period is shorter than about 40 days.

Several groups have already searched for close (sub)stellar companions of the radial-velocity $(R V)$ planet host stars using adaptive optics. However, an interesting regime of companions, with separations up to $\sim 1000 \mathrm{AU}$, is not accessible to those searches because of their small field of view (FOV). By using relatively wide field images and going relatively deep (see Sect. 2), we are able to detect wide (sub)stellar companions which could not be found so far by the less sensitive all-sky IR surveys like $2 \mathrm{MASS}^{1}$ or DENIS ${ }^{2}$.

\footnotetext{
* Based on observations obtained on La Silla in ESO programs 68.C-008 and 70.C-0116(A).

1 2MASS: 2 Micron All Sky Survey.

2 DENIS: Deep Near Infrared Survey of the Southern Sky.
}

Therefore, at the end of 2000, we started an observing program to search for unknown wide (sub)stellar companions of all stars known to harbor giant planets. So far, we have obtained a first image for most of our target stars with the $3.8 \mathrm{~m} \mathrm{UKIRT}^{3}$

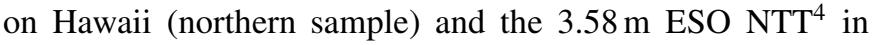
Chile (southern sample). In most cases the sensitivity of the IR cameras is sufficient to detect substellar companions with a separation down to the seeing limit $\left(\sim 1^{\prime \prime}\right)$. This implies that we are sensitive to companions with projected separations from $\sim 100$ AU up to several $1000 \mathrm{AU}$. For young $R V$ planet host stars like $\iota$ Hor (HD 17051) or $\epsilon$ Eri (HD 22049), with an age of only a few tens to a hundred Myrs, even wide planetary companions can be detected. The sensitivity is achieved by an observing strategy that avoids saturation close to the host star ( $\rightarrow$ detection of close companions) and by using relatively large array IR detectors ( $\rightarrow$ large FOV of more than 100 arcsec) for the detection of wide companions.

Our effort already yielded one new astrometric confirmation in the northern sample. We could detect common proper motion of the star HD 89744 and of a companion (Mugrauer et al. 2004 AN submitted), suggested by Wilson et al. (2001). The companion is separated by about $2500 \mathrm{AU}$ from its host star, with an effective temperature $\left(T_{\text {eff }}\right)$ of about $2200 \mathrm{~K}$ and a mass between 0.072 and $0.081 M_{\odot}$, depending on the evolutionary model and the assumed age. This companion to the

\footnotetext{
${ }^{3}$ UKIRT: United Kingdom InfraRed Telescope.

${ }^{4}$ NTT: New Technology Telescope.
} 
$R V$ host star, HD $89744 \mathrm{~B}$, is either a very low mass stellar object or a heavy brown dwarf companion to an $R V$ planet host star.

In this paper we report astrometric and spectroscopic evidence for a new stellar companion found in our southern survey of the region around the G0V star HD 75289, for which Udry et al. (2000) found a planet with $m \sin i=0.42 M_{\text {Jup }}$ in a 3.51 day orbit.

\section{Imaging, data reduction and calibration}

Our own observations of HD 75289 were obtained in the $H$ band $(1.6 \mu \mathrm{m})$ with the $3.58 \mathrm{~m}$ ESO NTT. This telescope is equipped with active optics which dramatically reduced dome and telescope seeing, yielding images with the seeing limit of the atmosphere. The IR detector is $\mathrm{SofI}^{5}$, a $1024 \times$ $1024 \mathrm{HgTeCd}$ detector with $18 \mu \mathrm{m}$ pixels and a pixel scale of approximately 0.144 arcsec in the so-called small field mode (147 arcsec FOV). To reduce saturation by the bright primary we chose an individual integration time to be as short as possible (1.2 s). To reach high sensitivity (i.e. a high limiting magnitude for the detection of faint companions), the total integration time was around $10 \mathrm{~min}$, composed of many short integrated images.

The auto-jitter technique of the NTT telescope was applied to delete the IR sky background from each raw frame. The datareduction was done with the ESO pipeline ECLIPSE ${ }^{6}$. All images were flat fielded with a special dome flat image, provided by the NTT science team. At 1 arcsec seeing the detection limit $(S / N=3)$ is $19 \mathrm{mag}$ in $H$ for a total integration time of $10 \mathrm{~min}$.

For calibration we identified 2MASS objects also detected on our NTT images. We used the coordinates of those objects from the 2MASS point source catalog to determine the NTT pixel scale. We did so on each NTT image and obtain the mean pixel scale for each NTT run. The averaged pixel scale of all runs is $143.66 \pm 0.15$ mas (only $0.1 \%$ relative uncertainty). With the pixel scale for each run, we could determine the positional difference (separation) between any two stars, for the 1 st and 2 nd epoch. The separations between non-moving background stars do not change with time. Using those non-moving background stars, we could then determine the proper motion of stars moving through the field. The precision of this method depends on the precision of Gaussian centering per star and on the number of the stars used. One can achieve $\sim 1 / 100$ of a pixel with Gaussian centering and special care (e.g. Pravdo \& Shaklan 1996). In our study, we have achieved a precision of $\sim 1 / 10$ of a pixel ( $\sim 20$ mas), good enough for measuring the proper motions of our relatively nearby target stars and their co-moving companions.

\section{Astrometry}

In our search of wide (sub)stellar companions we have to examine hundreds of faint objects close to the $R V$ planet host stars.

\footnotetext{
5 SofI: Son of Isaac.

${ }^{6}$ ECLIPSE: ESO C Library for an Image Processing Software Environment.
}

Most of those objects will prove to be ordinary background stars, randomly located close to, but far behind the target stars. On the other hand, bound companions share the proper motion of the host stars. This is so because the orbital motions of wide companions with separations $\geq 100$ AU are small compared to their much higher common proper motions. An astrometric survey will find these co-moving companions with only 2 images taken with some epoch difference, depending on the astrometric accuracy and the proper motion of the primary stars. Hence astrometry is a very effective tool for companion searches.

In a first step in our study of HD 75289 we compared our first epoch NTT image with the 2MASS one. The proper motion of objects that are bright enough can be derived by comparing their position in the 2MASS and the NTT images. The 2MASS images are accurate enough for the detection of comoving companions, as the proper motion of HD 75289 is large enough.

The proper motion of most stars, as derived from the 2MASS/NTT astrometry and the given epoch difference of $2.9 \mathrm{yr}$, were very small. Only one star had large proper motion, $\mu_{\alpha}=1 \pm 24$ mas and $\mu_{\delta}=-236 \pm 22$ mas per annum, consistent with the well known Hipparcos proper motion of HD 75289 $\left(\mu_{\alpha}=-20.50 \pm 0.49 \mathrm{mas} / \mathrm{yr}\right.$ and $\left.\mu_{\delta}=-227.68 \pm 0.44 \mathrm{mas} / \mathrm{yr}\right)$. It is clear that this star is a co-moving companion of the $R V$ planet host star. We therefore denote this star as HD 75289 B.

However, the 2MASS limit is approximately $2.5 \mathrm{mag}$ brighter than the NTT limit, hence the motion of the faint companion candidates could be investigated only with a second epoch NTT observation (see 2MASS and SofI/NTT image in Fig. 1).

Due to the large number of stars in the NTT FOV (see Fig. 1) several non-moving background stars were detected and the proper motion could be determined with a precision of the order of $\sim 20$ mas (see Fig. 2). Due to PSF saturation the proper motion of HD 75289 A couldn't be measured accurately in both NTT images, but could be calculated for the given epoch difference from Hipparcos data for the stellar parallax, yearly proper motion and equatorial coordinates (square in Fig. 2).

In addition we illustrate the proper motion of HD $75289 \mathrm{~B}$ over all three epochs with two reference stars R1 and R2 (see Figs. 1 and 3).

\section{Photometry}

Table 1 gives the apparent magnitude of HD 75289 A and B in $J H K_{\mathrm{S}}$ derived by $2 \mathrm{MASS}$, together with our derivation of $H_{\text {Sofi }}=11.224 \pm 0.040$. Our result is consistent with the 2MASS photometry. From the known spectral type of HD 75289 A and, hence, its expected intrinsic $B-V$ color $(B-V=0.58$ from Kenyon \& Hartmann 1995) and its published $B-V$ color (from Hipparcos $B-V=0.578 \pm 0.003 \mathrm{mag}$ ), we find that interstellar absorption is negligible, as expected for nearby stars. With $J-K$ from 2 MASS and color to temperature conversion from Kenyon \& Hartmann (1995) we can derive a $T_{\text {eff }}$ of between 5800 and $6380 \mathrm{~K}(J-K=0.355 \pm 0.029)$ for the primary, which is consistent with its published spectral type G0V (Udry et al. 2000). We obtained 3210 to $3860 \mathrm{~K}$ $(J-K=0.907 \pm 0.047)$ for the companion, hence a spectral 


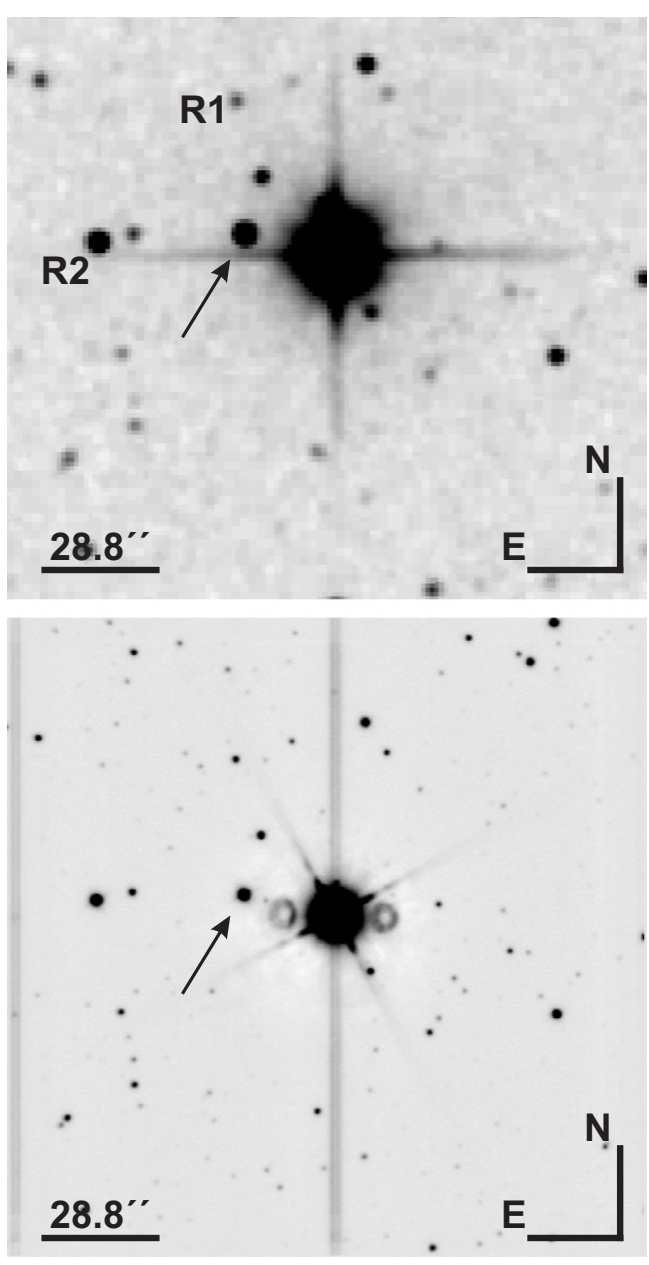

Fig. 1. $H$ band images of HD 75289 (central bright star) from 2MASS (02/99) (top) and our first epoch NTT/SofI image (01/02) (bottom). The total integration time is $10 \mathrm{~min}$. The co-moving companion is located 21.5 arcsec east of HD 75289 and is also visible in the 2MASS image (marked object). The stars R1 and R2 are used as comparison stars in Fig. 4.

Table 1. Photometry for HD $75289 \mathrm{~A}$ and B. The 2MASS Point Source catalog yield apparent $J H K_{\mathrm{S}}$ magnitudes which are confirmed in $H$ with our SofI/NTT images.

\begin{tabular}{l|c|c}
\hline \hline Band & $m_{A}$ & $m_{B}$ \\
\hline$J$ & $5.346 \pm 0.019$ & $11.750 \pm 0.036$ \\
$H$ & $5.187 \pm 0.031$ & $11.181 \pm 0.031$ \\
$H_{\text {Sofi }}$ & - & $11.224 \pm 0.040$ \\
$K_{\mathrm{S}}$ & $5.012 \pm 0.020$ & $10.879 \pm 0.027$ \\
\hline
\end{tabular}

type between M 0 and M 5. We used the 2MASS color transformations of Carpenter (2001) to convert $J-K_{\mathrm{S}}$ from 2MASS to $J-K$ of Bessel \& Brett which is similar to Johnson.

\section{Spectroscopy}

To confirm the spectral type of the companion we obtained IR spectra of HD 75289 A and B in June 2003 with SofI in spectroscopic mode. We used long slit spectroscopy with a slit width of one arcsec, and the red grism covering the wavelength

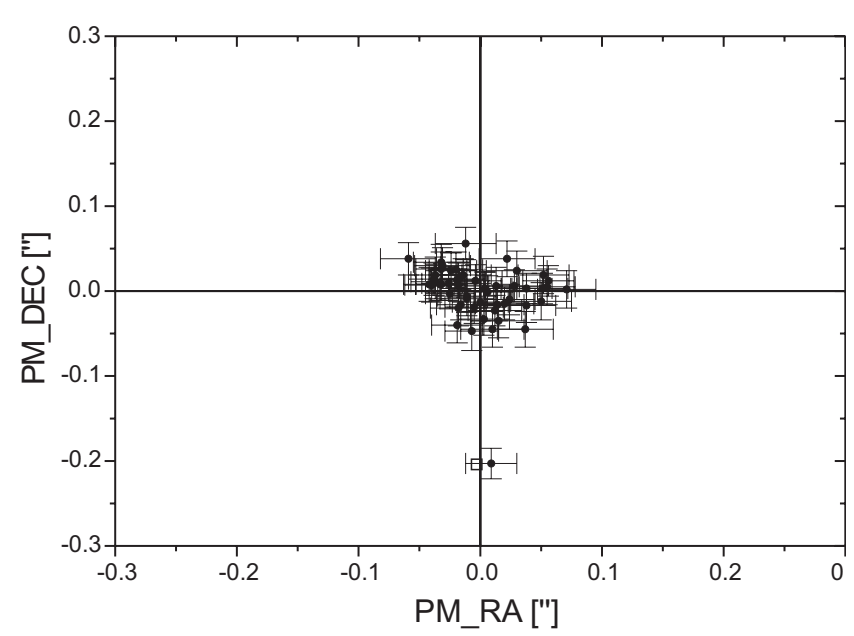

Fig. 2. Result of the astrometry obtained by comparing two NTT images from epochs $01 / 02$ and $12 / 02$. The formal proper motions of all detected objects around HD 75289 A are shown in the diagram. All of them have negligible proper motions, similar in size to the astrometric uncertainty ( 20 mas) hence they are very slowly moving background objects. Only HD 75289 B (bottom with error bars) shares the proper motion of HD 75289 A (square) which is well known from the Hipparcos astrometry. The proper motion of the bright primary star A is not measured in our NTT images because of saturation.

range from 1.53 to $2.52 \mu \mathrm{m}$. The dispersion was $10.22 \AA$ per pixel with an IR $\mathrm{HgCdTe}$ detector in the large field mode (288 mas pixel scale). The resolving power is $\lambda / \Delta \lambda \approx 588$.

Background subtraction was obtained by nodding between two positions along the slit, as well as by a small jitter around those two positions, to avoid individual pixels always seeing the same part of the sky. Eighteen individual spectra, each with an integration time of $30 \mathrm{~s}$, were averaged, giving a total integration time of $9 \mathrm{~min}$. All images were flat fielded with a standard dome flat and wavelength calibrated with a Xe lamp. We used standard IRAF routines for background subtraction, flat fielding and averaging all individual spectra.

The companion and the primary star were both located on the slit, and spectra of both objects were taken simultaneously. $T_{\text {eff }}$ of HD 75289 A is well known, hence a black body function with the given $T_{\text {eff }}(6030 \mathrm{~K})$ can be used to determine the response function of the spectrograph, which is needed to obtain relative flux calibrated spectra of both objects. In Fig. 4 we show the relative flux calibrated spectra of HD 75289 A and B. The continuum of the companion is much flatter than the primary continuum, consistent with a cooler photosphere. From a black body fit of the continuum of HD 75289 B we determine its $T_{\text {eff }}$ to be in the range between $3250 \mathrm{~K}$ and $3500 \mathrm{~K}$, hence its spectral type is M 2 to M 5.

Figure 5 shows the normalized $H$ and $K$ band spectra of HD $75289 \mathrm{~B}$. The most striking luminosity-sensitive feature in the $H$ band is the second-overtone $\mathrm{CO}$ band head $\left[v, v^{\prime}\right]$ at $6177 \mathrm{~cm}^{-1}$, which is found in the spectra of $\mathrm{K}$ and $\mathrm{M}$ stars. The spectrum of HD 75289 B shows CO molecular lines which are fainter than $\mathrm{Mg}\left(5844 \mathrm{~cm}^{-1}\right)$, typical for red dwarfs and in agreement with the $J H K$ absolute magnitudes derived in Sect. 4. The hydrogen line at $5950 \mathrm{~cm}^{-1}$, which can be found only in stars earlier than $\mathrm{K} 3$, is not visible in the companion 

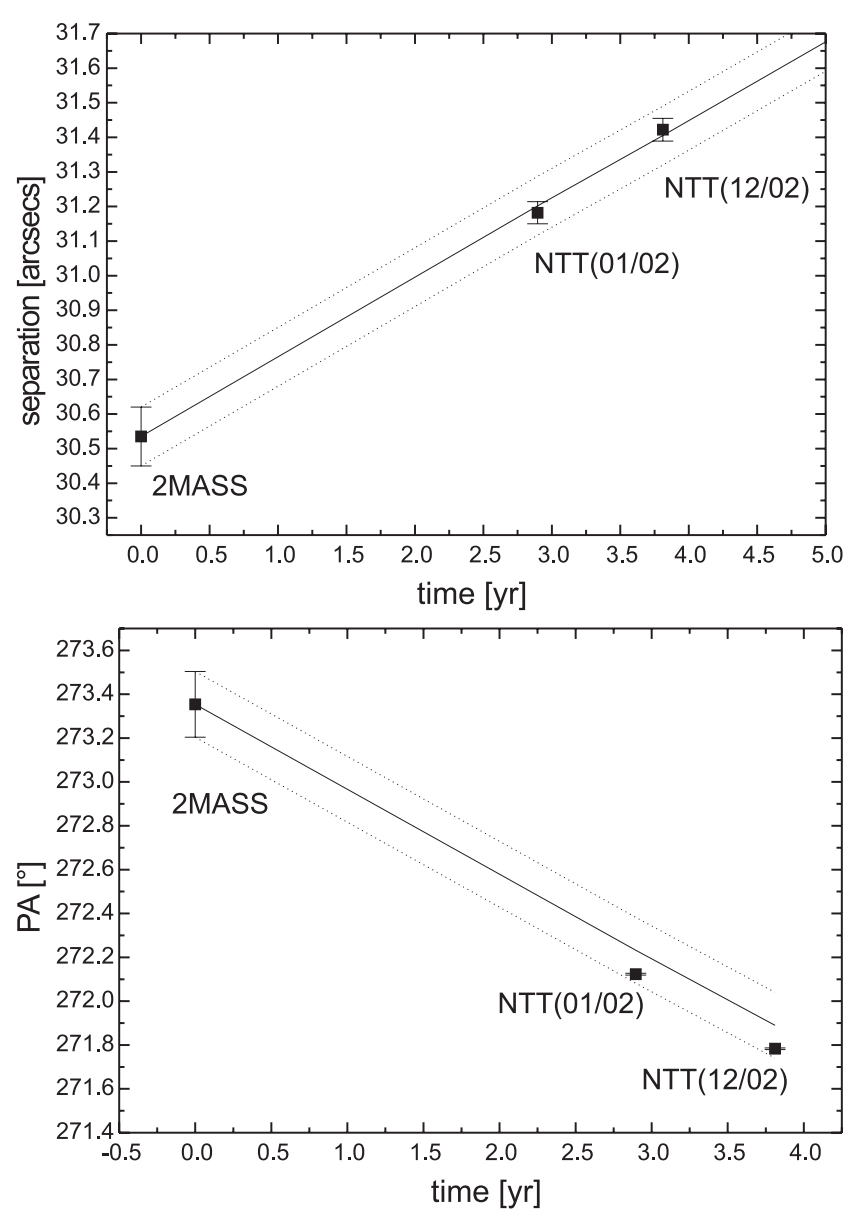

Fig. 3. Proper motion of HD 75289 B for all three epochs. We measure the distance between R1 and HD75289 B (top) and the position angle PA of HD 75289 B measured from R2. See Fig. 1 for the stars R1 and R2. Due to the motion of HD $75289 \mathrm{~B}$ relative to the reference stars both values are changing following the predicted curves (straight lines) for a co-moving companion to HD $752898 \mathrm{~A}$. The astrometric uncertainty is illustrated with dotted lines.

spectrum and the $\mathrm{Al}$ feature at $5973 \mathrm{~cm}^{-1}$ is as strong as the $\mathrm{Mg}$ line. Both comparisons serve as evidence for a spectral type cooler than M1V. The Si line at $6264 \mathrm{~cm}^{-1}$ is not apparent and $\mathrm{Si}$ at $6292 \mathrm{~cm}^{-1}$ is faint as are the the $\mathrm{OH}(\Delta v=2)$ molecular features at $5920 \mathrm{~cm}^{-1}$, typical for a spectral type M3V.

The strongest lines in the spectrum in $\mathrm{K}$ of the companion are from molecular bands of the first $\mathrm{CO}$ overtone extending from $4360 \mathrm{~cm}^{-1}$ to the low frequency side of the spectrum. In addition, ${ }^{13} \mathrm{CO}$ at $4260 \mathrm{~cm}^{-1}$ and $\mathrm{H} \mathrm{Br} \gamma$ at $6297 \mathrm{~cm}^{-1}$ are not apparent, all argue for a dwarf cooler than $\mathrm{K} 2 \mathrm{~V}$. The $\mathrm{CO}$ bands are a bit stronger than the $\mathrm{Ca} / \mathrm{Fe}\left(4415 \mathrm{~cm}^{-1}\right)$ as the $\mathrm{Na}\left(4530 \mathrm{~cm}^{-1}\right)$ atomic features and $\mathrm{Ca} / \mathrm{Fe}$ is weaker than $\mathrm{Na}$. The Al line at $6720 \mathrm{~cm}^{-1}$ is faint but the $\mathrm{Mg}$ line at $4750 \mathrm{~cm}^{-1}$ is missing, typical for spectral types cooler than M3V.

The detected features in the spectrum of HD $75289 \mathrm{~B}$ in $H$ and $K$, the black body fit of the continuum, and the $J H K$ colors of the companion, are all consistent with a spectral type of M2V to M5V, i.e. $T_{\text {eff }}$ is in the range of 3240 to $3580 \mathrm{~K}$.

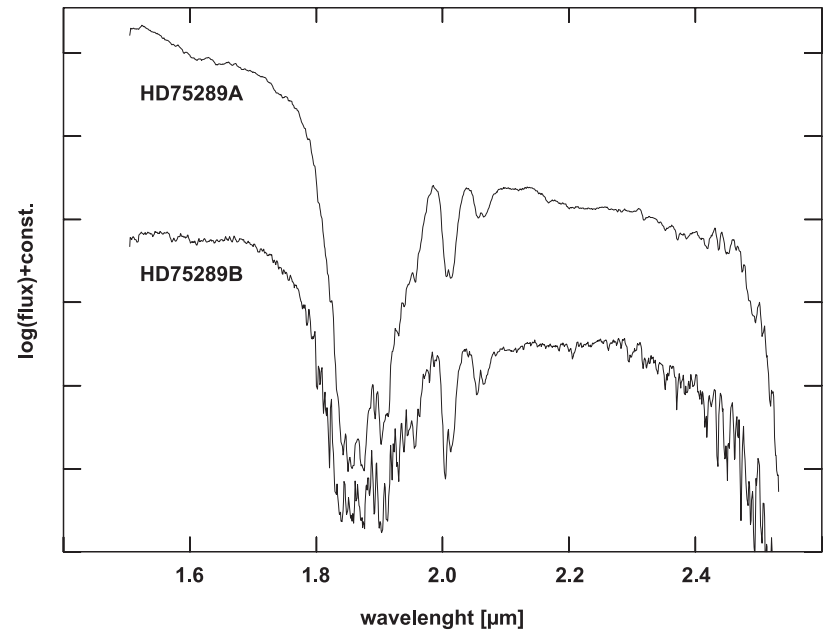

Fig. 4. Relative flux of HD $75289 \mathrm{~A}$ and B. The drop at $1.85 \mu \mathrm{m}$ is due to strong water absorption as well as some telluric lines. From a black body fit, we derive a $T_{\text {eff }}$ of the companion between $3250 \mathrm{~K}$ and $3500 \mathrm{~K}$, in agreement with a spectral type M 2 to M 5 .
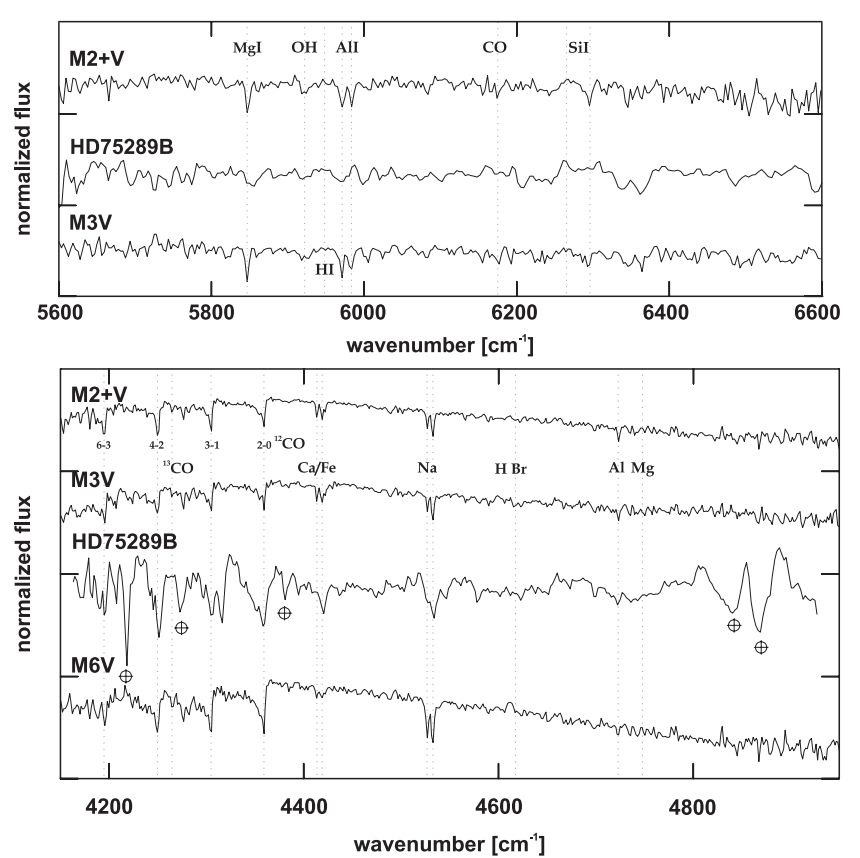

Fig. 5. Normalized $H$ and $K$ band spectra of HD 75289 B, compared with spectra of GJ 411 (M 2+V), GJ 725 (M3V) and Wolf 359 (M6V) from Meyer et al. (1997/98).

\section{Discussion}

HD 75289 is a bright G0 dwarf ( $V=6.36 \mathrm{mag})$ located at a distance of $28.94 \pm 0.47 \mathrm{pc}$ (distance modulus $2.308 \pm 0.036 \mathrm{mag}$ ). Its apparent $J H K$ colors are typical for a G0V star at the given distance. Thus, the super-giant classification given in Simbad is invalidated, as pointed out by Udry et al. (2000). The same group discovered an extrasolar planet with a minimum mass of $0.42 M_{\text {Jup }}$ which revolves around its parent star in a nearly circular orbit ( $e=0.054, a=0.046 \mathrm{AU})$.

HD 75289 B is clearly co-moving with HD 75289 A and the color-magnitude relation agrees with the assumption that both 


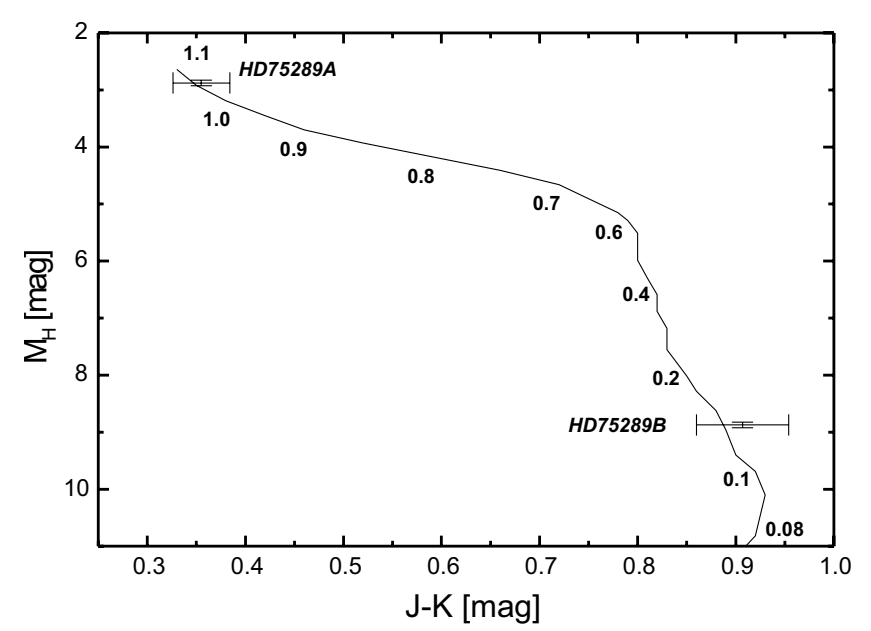

Fig. 6. The color-magnitude diagram with the isochrone for $5 \mathrm{Gyr}$ from Baraffe et al. (1998) with $[\mathrm{M} / \mathrm{H}]=0$, mixing length parameter $\alpha=1$ and $\mathrm{He}$ abundance $Y=0.25$. The primary and its companion are included in the diagram with their uncertainties in magnitude and color. Masses are indicated as numbers in solar masses.

objects are at the same distance (Fig. 6). With Baraffe et al. (1998) models, the JHK colors from Sect. 3 and the given distance modulus, we can derive the mass of HD75289 B to be $0.135 \pm 0.003 M_{\odot}$, see Fig. 6 . The system age is roughly $5 \mathrm{Gyr}$ (Udry et al. 2000). Note that the age uncertainty of the primary does not play an important role in the derivation of the mass of HD $75289 \mathrm{~B}$, because the IR magnitudes for such low-mass stellar objects decrease very slowly from 1 to $10 \mathrm{Gyr}$. The given uncertainty of the companion mass is derived form the magnitude errors only. Inaccuracies of the used theoretical model were not considered here.

With the derived companion mass $\left(0.135 M_{\odot}\right)$, the primary mass $\left(\sim 1 M_{\odot}\right)$ and the companion separation $621 \mathrm{AU}(21.465 \pm$ $0.023 \mathrm{arcsec}$ ) we can compute the expected $R V$ variation of the primary induced by the presence of the wide companion $v \sim$ $150 \mathrm{~m} / \mathrm{s}$ with an orbital period of $\sim 15000$ years. Although this is a large effect, the maximal yearly variation of the $R V$ is only $\sim 0.07 \mathrm{~m} / \mathrm{s}$, too small to be detected in the foreseeable future.

Figure 7 shows the NTT detection limit which is 19 mag in $H$ and enables the detection of substellar companions down to $M_{H}=16.7 \mathrm{mag}$ around HD 75289 A ( $m \geq 44 M_{\text {Jup }}$ according to Baraffe et al. 2003). Objects at distances of up to 68 arcsec were observed twice but no further co-moving companion could be identified. Further stellar companions ( $m \geq 75 M_{\text {Jup }}$ ) can be ruled out for a projected separation from $136 \mathrm{AU}$ up to $1968 \mathrm{AU}$

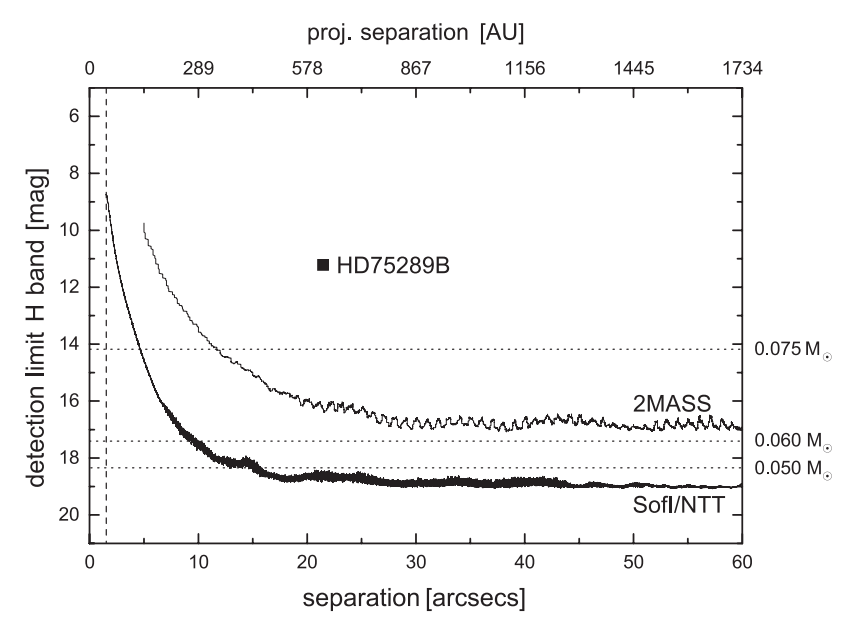

Fig. 7. The limiting $H$ magnitude versus separation from HD 75289 A for our NTT image shown in Fig. 1 and 2MASS. The corresponding projected separation in AU is shown on the upper $x$-axis. Saturation occurs within $1.5 \operatorname{arcsec}(43 \mathrm{AU})$ (see vertical dashed line) hence a companion search is impossible there. The detection of all stellar companions is feasible beyond $4.7 \operatorname{arcsec}(136 \mathrm{AU})$. The right $y$-scale shows the predicted absolute $H$ magnitudes for substellar objects from Baraffe et al. (2003) models for an age of 5 Gyr. The $3 \sigma$ detection limit is $19 \mathrm{mag}$ in $H$, hence substellar companions down to $0.050 M_{\odot}$ can be found beyond $15 \operatorname{arcsec}(434 \mathrm{AU})$.

Acknowledgements. We would like to thank the technical staff of the ESO NTT for all their help and assistance in carrying out the observations. Furthermore, we would like to thank M. Fernández, A. Seifahrt, A. Szameit and C. Broeg who have carried out some of the observations of this project. We made use of the 2MASS public data releases as well as the Simbad database operated at the Observatoire Strasbourg. This work was partly supported by the Israel Science Foundation (grant No. 233/03)

\section{References}

Baraffe, I., Chabrier, G., \& Allard, F. 1998, A\&A, 337, 403

Baraffe, I., Chabrier, G., \& Barman, T. S. 2003, A\&A, 402, 701

Carpenter, J. M. 2001, AJ, 121, 2851

Eggenberger, A., Udry, S., \& Mayor, M. 2004, A\&A, 417, 353

Gonzales, G., \& Laws, C. 2000, AJ, 119, 390

Kenyon, S. J., \& Hartmann, L. W. 1995, ApJS, 101, 117

Meyer, M. R., Edwards, S., \& Hinkle, K. H. 1998, ApJ, 508, 397

Pravdo Steven, H., \& Shaklan Stuart, B. 1996, ApJ, 465, 264

Udry, S., Mayor, M., \& Naef, D. 2000, A\&A, 356, 590

Wallace, L., \& Hinkle, K. 1997, ApJS, 111, 445

Wilson, J. C., Kirkpatrick, J. D., \& Gizis, J. E. 2001, AJ, 122, 1989

Zucker, S., \& Mazeh, T. 2002, ApJ, 568, 113 